\title{
Front Matter: Volume 7600
}

, "Front Matter: Volume 7600," Proc. SPIE 7600, Ultrafast Phenomena in Semiconductors and Nanostructure Materials XIV, 760001 (1 April 2010); doi: $10.1117 / 12.862672$

SPIE. Event: SPIE OPTO, 2010, San Francisco, California, United States 


\title{
PROCEEDINGS OF SPIE
}

\section{Ultrafast Phenomena in Semiconductors and Nanostructure Materials XIV}

\author{
Jin-Joo Song \\ Kong-Thon Tsen \\ Markus Betz \\ Abdulhakem Y. Elezzabi \\ Editors
}

24-27 January 2010

San Francisco, California, United States

Sponsored and Published by

SPIE

Volume $\mathbf{7 6 0 0}$ 
The papers included in this volume were part of the technical conference cited on the cover and title page. Papers were selected and subject to review by the editors and conference program committee. Some conference presentations may not be available for publication. The papers published in these proceedings reflect the work and thoughts of the authors and are published herein as submitted. The publisher is not responsible for the validity of the information or for any outcomes resulting from reliance thereon.

Please use the following format to cite material from this book:

Author(s), "Title of Paper," in Ultrafast Phenomena in Semiconductors and Nanostructure Materials $X I V$, edited by Jin-Joo Song, Kong-Thon Tsen, Markus Betz, Abdulhakem Y. Elezzabi, Proceedings of SPIE Vol. 7600 (SPIE, Bellingham, WA, 2010) Article CID Number.

ISSN 0277-786X

ISBN 9780819479969

Published by

SPIE

P.O. Box 10, Bellingham, Washington 98227-0010 USA

Telephone +1 3606763290 (Pacific Time) · Fax +1 3606471445

SPIE.org

Copyright (C) 2010, Society of Photo-Optical Instrumentation Engineers

Copying of material in this book for internal or personal use, or for the internal or personal use of specific clients, beyond the fair use provisions granted by the U.S. Copyright Law is authorized by SPIE subject to payment of copying fees. The Transactional Reporting Service base fee for this volume is $\$ 18.00$ per article (or portion thereof), which should be paid directly to the Copyright Clearance Center (CCC), 222 Rosewood Drive, Danvers, MA 01923. Payment may also be made electronically through CCC Online at copyright.com. Other copying for republication, resale, advertising or promotion, or any form of systematic or multiple reproduction of any material in this book is prohibited except with permission in writing from the publisher. The CCC fee code is 0277-786X/10/\$18.00.

Printed in the United States of America.

Publication of record for individual papers is online in the SPIE Digital Library.

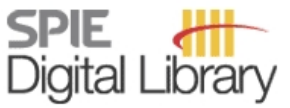

SPIEDigitalLibrary.org

Paper Numbering: Proceedings of SPIE follow an e-First publication model, with papers published first online and then in print and on CD-ROM. Papers are published as they are submitted and meet publication criteria. A unique, consistent, permanent citation identifier (CID) number is assigned to each article at the time of the first publication. Utilization of CIDs allows articles to be fully citable as soon they are published online, and connects the same identifier to all online, print, and electronic versions of the publication. SPIE uses a six-digit CID article numbering system in which:

- The first four digits correspond to the SPIE volume number.

- The last two digits indicate publication order within the volume using a Base 36 numbering system employing both numerals and letters. These two-number sets start with $00,01,02,03,04$, $05,06,07,08,09,0 A, 0 B \ldots 0 Z$, followed by 10-1Z, 20-2Z, etc.

The CID number appears on each page of the manuscript. The complete citation is used on the first page, and an abbreviated version on subsequent pages. Numbers in the index correspond to the last two digits of the six-digit CID number. 


\section{Contents}

ix Conference Committee

\section{KEYNOTE SESSION}

760002 Femtosecond quantum optics with semiconductor nanostructures: single cycles of light, electrons, and photons [7600-01]

A. Leitenstorfer, R. Huber, R. Bratschitsch, Univ. Konstanz (Germany)

\section{EXCITONS AND PHONONS}

760008 Two-photon absorption and multi-exciton generation in lead salt quantum dots [7600-07] L. A. Padilha, CREOL \& FPCE, The College of Optics and Photonics, Univ. of Central Florida (United States); G. Nootz, CREOL \& FPCE, The College of Optics and Photonics, Univ. of Central Florida (United States) and Univ. of Central Florida (United States); S. Webster, CREOL \& FPCE, The College of Optics and Photonics, Univ. of Central Florida (United States); D. J. Hagan, E. W. Van Stryland, CREOL \& FPCE, The College of Optics and Photonics, Univ. of Central Florida (United States) and Univ. of Central Florida (United States); L. Levina, V. Sukhovatkin, E. H. Sargent, Univ. of Toronto (Canada)

\section{CARRIER DYNAMICS}

7600 OB Transient optical gain and carrier dynamics in Ge/SiGe quantum wells (Invited Paper) [7600-10]

N. S. Köster, C. Lange, S. Chatterjee, Philipps-Univ. Marburg (Germany); H. Sigg, Paul Scherrer Institut (Switzerland); D. Chrastina, G. Isella, Politecnico di Milano (Italy); H. von Känel, EpiSpeed AG (Switzerland); M. Schäfer, M. Kira, S. W. Koch, Philipps-Univ. Marburg (Germany)

7600 OD Ultrafast transient absorption studies of single metal and semiconductor nanowires (Invited Paper) [7600-12]

G. V. Hartland, C. R. Carey, H. Staleva, Univ. of Notre Dame (United States)

7600 OE Fast carrier dynamics in new GaAs deep-center laser for $1.3 \mu \mathrm{m}-1.5 \mu \mathrm{m}$ fiber optics (Invited Paper) [7600-13]

J. L. Pan, Yale Univ. (United States)

\section{THz SPECTROSCOPY AND APPLICATIONS I}

7600 OF Ultrafast terahertz response of optically excited semiconductor heterostructures (Invited Paper) [7600-14]

D. Golde, M. Kira, S. W. Koch, Philipps-Univ. Marburg (Germany) 
7600 OG Recent progress on efficient generation of monochromatic THz pulses based on difference-frequency generation (Invited Paper) [7600-15]

Y. J. Ding, Y. Jiang, G. Xu, Lehigh Univ. (United States); I. B. Zotova, ArkLight, Inc. (United States)

$7600 \mathrm{OH}$ Time-resolved terahertz spectroscopy of conjugated polymer/CdSe nanorod composites (Invited Paper) [7600-16]

D. G. Cooke, Technical Univ. of Denmark (Denmark); J. Y. Lek, Nanyang Technological Univ. (Singapore); F. C. Krebs, Risø National Lab. (Denmark); Y. M. Lam, Nanyang Technological Univ. (Singapore); P. U. Jepsen, Technical Univ. of Denmark (Denmark)

7600 ol Ultrafast terahertz spectroscopy for measuring carrier dynamics in nanoscale photovoltaic materials (Invited Paper) [7600-17]

O. Esenturk, National Institute of Standards and Technology (United States); P. A. Lane, J. S. Melinger, U.S. Naval Research Lab. (United States); E. J. Heilweil, National Institute of Standards and Technology (United States)

7600 0J THz spectroscopy as a new tool to probe hydration dynamics (Invited Paper) [7600-18] M. Havenith, Ruhr-Univ. Bochum (Germany)

PLASMONICS I

7600 OM Evidence of terahertz emission from a particle plasmon Schottky barrier (Invited Paper) [7600-21]

C. J. E. Straatsma, C. A. Baron, A. Y. Elezzabi, Univ. of Alberta (Canada)

SPINS AND SPINTRONICS I

7600 OR Ultrafast dynamics and optical spin-control in single magnetic quantum dots (Invited Paper) [7600-26]

V. M. Axt, Univ. Bayreuth (Germany); D. E. Reiter, T. Kuhn, Westfälische Wilhelms-Univ. Münster (Germany)

7600 OS Microscopic theoretical analysis of optically generated injection currents in semiconductor quantum wells [7600-27]

H. T. Duc, J. Förstner, T. Meier, Univ. Paderborn (Germany)

7600 OT Spin dynamics and manipulation in GaMnAs alloys (Invited Paper) [7600-28]

X. Liu, Y. Y. Zhou, Univ. of Notre Dame (United States); E. Harley, L. E. McNeil, Univ. of North Carolina at Chapel Hill (United States); J. Wang, lowa State Univ. (United States); J. Qi, Y. Xu, A. Steigerwald, N. Tolk, Vanderbilt Univ. (United States); J. P. Zahn, A. Gamouras, S. March,

K. C. Hall, Dalhousie Univ. (Canada); J. K. Furdyna, Univ. of Notre Dame (United States)

NONLINEAR OPTICAL PHENOMENA I

7600 OU Probing many-body interactions in a disordered semiconductor quantum well with electronic two-dimensional Fourier transform spectroscopy (Invited Paper) [7600-29] Z. Sun, T. W. Jarvis, X. Li, The Univ. of Texas at Austin (United States); M. Erementchouk, M. N. Levenberger, Univ. of Central Florida (United States) 
7600 0X Temporal femtosecond pulse tailoring for nanoscale laser processing of wide-bandgap materials (Invited Paper) [7600-32]

M. Wollenhaupt, L. Englert, A. Horn, T. Baumert, Univ. Kassel (Germany)

\section{NANOSTRUCTURES AND NANOPHOTONICS II}

7600 OY Ultrafast optical control of electron spins in quantum wells and quantum dots (Invited Paper) [7600-33]

S. G. Carter, S. E. Economou, Naval Research Lab. (United States); A. Shabaev, George Mason Univ. (United States); T. A. Kennedy, A. S. Bracker, T. L. Reinecke, Naval Research Lab. (United States); Z. Chen, S. T. Cundiff, JILA (United States)

760010 Enhancement of optical emission and absorption by metal nanoparticles (Invited Paper) [7600-35]

G. Sun, Univ. of Massachusetts, Boston (United States); J. B. Khurgin, The Johns Hopkins Univ. (United States)

\section{PLASMONICS II}

760013 Ultrafast silicon-plasmonic modulators [7600-38]

S. Sederberg, Z. Han, V. Vien, A. Y. Elezzabi, Univ. of Alberta (Canada)

\section{SPECIAL TOPICS}

760015 Up on the Jaynes-Cummings ladder of an exciton-cavity system (Invited Paper) [7600-44] J. Kasprzak, Cardiff Univ. (United Kingdom); S. Reitzenstein, Univ. Würzburg (Germany);

E. A. Muljarov, Cardiff Univ. (United Kingdom); C. Kistner, C. Schneider, M. Strauss, S. Höfling, A. Forchel, Univ. Würzburg (Germany); W. Langbein, Cardiff Univ. (United Kingdom)

760017 New phenomena in interaction of intense ultrashort light pulses with transparent materials: from 3D self-assembled nanostructures to quill writing and nonreciprocal photosensitivity (Invited Paper) [7600-41]

P. G. Kazansky, M. Beresna, Univ. of Southampton (United Kingdom); Y. Shimotsuma, K. Hirao, Kyoto Univ. (Japan); Y. P. Svirko, Univ. of JoensuU (Finland)

760019 Development and application of plasma waveguide based soft x-ray lasers (Invited Paper) [7600-43]

J. Wang, Institute of Atomic and Molecular Sciences (Taiwan), National Taiwan Univ.

(Taiwan), and National Central Univ. (Taiwan); S. Chen, Institute of Atomic and Molecular Sciences (Taiwan) and National Central Univ. (Taiwan); J.-Y. Lin, National Chung Cheng Univ. (Taiwan); H.-H. Chu, National Central Univ. (Taiwan); M.-C. Chou, Institute of Atomic and Molecular Sciences (Taiwan) and National Central Univ. (Taiwan); P.-H. Lin, Institute of Atomic and Molecular Sciences (Taiwan), National Taiwan Univ. (Taiwan), and National Central Univ. (Taiwan) 
7600 1B Proposal for electrical detection of spin separation with in-plane magnetic field in mesoscopic Stern-Gerlach spin filter [7600-46]

M. Kohda, Tohoku Univ. (Japan) and Japan Science and Technology Agency (Japan); J. Ohe, Tohoku Univ. (Japan); H. Sanada, NTT Basic Research Labs. (Japan); M. Yamamoto, National Institute for Materials Science (Japan); T. Ohtsuki, Sophia Univ. (Japan); J. Nitta, Tohoku Univ. (Japan)

7600 1D Electronic dynamics due to exchange interaction with holes in GaAs (Invited Paper) [7600-48]

H. C. Schneider, M. Krauß, Univ. Kaiserslautern (Germany)

\section{NONLINEAR OPTICAL PHENOMENA II}

7600 IE Advances in optical two-dimensional spectroscopy applied to the study of semiconductor and atomic systems (Invited Paper) [7600-49]

A. D. Bristow, X. Dai, D. Karaiskaj, G. A. Moody, S. T. Cundiff, JILA (United States)

7600 IF Exciton annihilation and dephasing dynamics in semiconducting single-walled carbon nanotubes (Invited Paper) [7600-50]

M. W. Graham, Univ. of California, Berkeley (United States); Y.-Z. Ma, Univ. of California, Berkeley (United States) and Oak Ridge National Lab. (United States); A. A. Green,

M. C. Hersam, Northwestern Univ. (United States); G. R. Fleming, Univ. of California, Berkeley (United States)

$76001 G$ Probing many particle correlations in semiconductor quantum wells using doublequantum-coherence signal (Invited Paper) [7600-51]

L. Yang, S. Mukamel, Univ. of Califorina, Irvine (United States)

$76001 \mathrm{H}$ Dynamics of shaping ultrashort optical dissipative solitary pulses in the actively mode-locked semiconductor laser with an external long-haul single-mode fiber cavity [7600-52]

A. S. Shcherbakov, P. Moreno Zarate, National Institute for Astrophysics, Optics, and Electronics (Mexico)

760011 Confined electron emission with femtosecond timing: nonlinearity, localization, enhancement (Invited Paper) [7600-53]

M. Gulde, R. Bormann, A. Weismann, S. V. Yalunin, C. Ropers, Georg-August-Univ. Göttingen (Germany)

\section{NONLINEAR OPTICAL PHENOMENA III}

$76001 \mathrm{~J} \quad$ Multiple-quantum 2D spectroscopy of many-body correlations in GaAs quantum wells (Invited Paper) [7600-54]

D. B. Turner, K. W. Stone, P. Wen, D. H. Arias, K. A. Nelson, Massachusetts Institute of Technology (United States) 
$76001 \mathrm{~K}$ Ultrafast coherent control of electric currents at metal surfaces (Invited Paper) [7600-55]

J. Güdde, M. Rohleder, Philipps-Univ. Marburg (Germany); T. Meier, Univ. Paderborn

(Germany); S. W. Koch, U. Höfer, Philipps-Univ. Marburg (Germany)

$7600 \mathrm{lL} \quad$ Two-dimensional mode-locking in planar waveguide arrays [7600-56]

M. O. Williams, J. N. Kutz, Univ. of Washington (United States)

$76001 \mathrm{M}$ Toward nonlinear magneto-optics: collapse detuning via magnetically adjustable linear and circular birefringences (Invited Paper) [7600-57]

K. A. Rutkowska, INRS-Énergie et Matériaux, Univ. du Québec (Canada) and Warsaw Univ. of Technology (Poland); Y. Linzon, INRS-Énergie et Matériaux, Univ. du Québec (Canada); B. A. Malomed, Tel Aviv Univ. (Israel); R. Morandotti, INRS-Énergie et Matériaux, Univ. du Québec (Canada)

\section{NANOSTRUCTURES AND NANOPHOTONICS III}

7600 iN Manipulation of a single Mn spin using excitation transfer between two coupled CdTe/ZnTe quantum dots (Invited Paper) [7600-58]

M. Goryca, Univ. of Warsaw (Poland)

$76001 Q \quad$ Low-dimensional plasmons in atom-scale metallic objects (Invited Paper) [7600-61]

T. Nagao, National Institute for Materials Science (Japan)

\section{THz SPECTROSCOPY AND APPLICATIONS II}

7600 is Extreme THz nonlinearities in bulk and nanostructured semiconductors (Invited Paper) [7600-63]

A. Sell, A. A. Anappara, Univ. of Konstanz (Germany); T. Kampfrath, K. von Volkmann, M. Wolf, Freie Univ. Berlin (Germany); J. T. Steiner, M. Kira, S. W. Koch, Univ. Marburg (Germany); G. Biasiol, Lab. Nazionale TASC, CNR-INFM (Italy); L. Sorba, Lab. Nazionale TASC, CNR-INFM (Italy) and Lab. NEST, CNR-INFM, Scuola Normale Superiore di Pisa (Italy);

A. Tredicucci, Lab. NEST, CNR-INFM, Scuola Normale Superiore di Pisa (Italy); A. Leitenstorfer, R. Huber, Univ. of Konstanz (Germany)

7600 IT Nonlinear terahertz spectroscopy (Invited Paper) [7600-64]

M. Woerner, W. Kuehn, K. Reimann, T. Elsaesser, Max-Born-Institut für Nichtlineare Optik und Kurzzeitspektroskopie (Germany); R. Hey, Paul-Drude-Institut für Festkörperelektronik (Germany)

7600 IV Terahertz radiation emission from silicon and magnesium doped indium nitride (Invited Paper) [7600-66]

I. Wilke, Rensselaer Polytechnic Institute (United States)

\section{POSTER SESSION}

7600 IW Ultrafast conductivity dynamics in optically excited InGaN/GaN multiple quantum wells observed by transient THz spectroscopy [7600-67]

D. Turchinovich, H. P. Porte, D. G. Cooke, P. Und Jepsen, Technical Univ. of Denmark (Denmark) 
$76001 Y \quad$ Terahertz plasmonic imaging [7600-69]

P. Maraghechi, C. Straatsma, A. Y. Elezzabi, Univ. of Alberta (Canada)

Author Index

viii

Downloaded From: https://www.spiedigitallibrary.org/conference-proceedings-of-spie on 26 Apr 2023 Terms of Use: https://www.spiedigitallibrary.org/terms-of-use 


\title{
Conference Committee
}

\author{
Symposium Chair
}

E. Fred Schubert, Rensselaer Polytechnic Institute (United States)

Symposium Cochairs

Liang-Chy Chien, Kent State University (United States)

James G. Grote, Air Force Research Laboratory (United States)

Program Track Chair

James G. Grote, Air Force Research Laboratory (United States)

Conference Chairs

Jin-Joo Song, University of California, San Diego (United States)

Kong-Thon Tsen, Arizona State University (United States)

\section{Conference Cochairs}

Markus Betz, Technische Universität Dortmund (Germany)

Abdulhakem Y. Elezzabi, University of Alberta (Canada)

\section{Program Committee}

Mischa Bonn, FOM Institute for Atomic and Molecular Physics

(Netherlands)

Yujie J. Ding, Lehigh University (United States)

Jan A. Gaj, University of Warsaw (Poland)

Rupert Huber, Universität Konstanz (Germany)

Robert A. Kaindl, Lawrence Berkeley National Laboratory (United States)

Jacek Kasprzak, Cardiff University (United Kingdom)

Daisik Kim, Seoul National University (Korea, Republic of)

Torsten Meier, Universität Paderborn (Germany)

Walter Pfeiffer, Universität Bielefeld (Germany)

Chi-Kuang Sun, National Taiwan University (Taiwan)

Fabrice Vallee, Université Claude Bernard Lyon 1 (France)

Hailin Wang, University of Oregon (United States)

Klaas Wynne, University of Strathclyde (United Kingdom)

Chih-Chung Yang, National Taiwan University (Taiwan) 
Session Chairs

Keynote Session

Jin-Joo Song, University of California, San Diego (United States)

$1 \quad$ Nanostructures and Nanophotonics I

Jin-Joo Song, University of California, San Diego (United States)

Dai-Sik Kim, Seoul National University (Korea, Republic of)

2 Excitons and Phonons

Yujie J. Ding, Lehigh University (United States)

3 Carrier Dynamics

Torsten Meier, Universität Paderborn (Germany)

$4 \quad$ THz Spectroscopy and Applications I

Mischa Bonn, FOM Institute for Atomic and Molecular Physics (Netherlands)

5 Plasmonics 1

Stephan W. Koch, Philipps-Universität Marburg (Germany)

Sangam Chatterjee, Philipps-Universität Marburg (Germany)

6 Spins and Spintronics I

René Beigang, Fraunhofer-Institut für Physikalische Messtechnik (Germany)

$7 \quad$ Nonlinear Optical Phenomena I

Henry M. van Driel, University of Toronto (Canada)

$8 \quad$ Nanostructures and Nanophotonics II

Abdulhakem Y. Elezzabi, University of Alberta (Canada)

9 Plasmonics II

Markus Betz, Technische Universität Dortmund (Germany)

10 Special Topics

Kong-Thon Tsen, Arizona State University (United States)

11 Spins and Spintronics II

Vollrath Martin Axt, Universität Bayreuth (Germany)

12 Nonlinear Optical Phenomena II

Abdulhakem Y. Elezzabi, University of Alberta (Canada)

13 Nonlinear Optical Phenomena III

Alan D. Bristow, JILA (United States) 
14 Nanostructures and Nanophotonics III

Ulrich Höfer, Philipps-Universität Marburg (Germany)

$15 \mathrm{THz}$ Spectroscopy and Applications II

Markus Betz, Technische Universität Dortmund (Germany) 
Downloaded From: https://www.spiedigitallibrary.org/conference-proceedings-of-spie on 26 Apr 2023

Terms of Use: https://www.spiedigitallibrary.org/terms-of-use 